[116] G. C. Temes and D. A. Calahan [17].

[117] G. C. Temes and D. Y. F. Zai [79].

[118] A. D. Waren and L. S Lasdon, "Practical filter design using mathematical optimization," Proc. 3rd Allerton Conf. on Circuit and System Theory (Urbana, Ill.), pp. 677-689, October 1965.

[119] A. D. Waren, L. S. Lasdon, and D. F. Suchman [18].

\section{Miscellaneous}

[120] G. A. Bekey and W. J. Karplus, Hybrid Computation. New York: Wiley, 1968, ch. 9.

[121] G. C. Broyden, "A new method of solving nonlinear simultaneous equations," Computer J., vol. 12, pp. 94-99, February 1969.
[122] J. Chernak, "Hybrid computer techniques and applications," in System Analysis by Digital Computer, F. F. Kuo and J. F. Kaiser, Eds. New York: Wiley, 1966.

[123] H. W. Kuhn and A. W. Tucker, "Non-linear programming," Proc. 2nd Symp. on Math. Statistics and Probability. Berkeley, Calif.: University of California Press, 1951, pp. 481-493.

[124] M. R. Osborne and G. A. Watson, "An algorithm for minimax approximation in the nonlinear case," Computer J., vol. 12, pp. 63-68, February 1969.

[125] A. Ralston, "Rational Chebyshev approximation," in Mathematical Methods for Digital Computers, vol. 2, A. Ralston and H. S. Wilf, Eds. New York: Wiley, 1967.

[126] A. Wexler, "Computation of electromagnetic fields," this issue. pp. $416-439$

\title{
Optimization of Microwave Networks by Razor Search
}

\author{
JOHN W. BANDLER, MEMBER, IEEE, AND PATRICK A. MACDONALD
}

\begin{abstract}
A new optimization method called razor search is presented. The method, which is based on pattern search, was specifically developed for the automatic optimization by computer of networks for which the objective is to minimize the maximum deviation of some response from a desired ideal response specification. Minimax response objectives, which can lead to equal-ripple optima, will in general give rise to discontinuous partial derivatives of the objective function with respect to the network parameters. Otherwise efficient optimization methods may slow down or even fail to reach an optimum in such circumstances, particularly when the response hypersurface has a narrow curved valley along which the path of discontinuous derivatives lies. Another direct search method called ripple search is also presented. This method was developed to locate the extrema of multimodal functions of one variable in an efficient manner, and is used to determine the maximum deviation of the response from the desired response. Sufficiently detailed flow diagrams are available so that the methods can be readily programmed. The razor search strategy (with ripple search) has been successfully applied to the optimization of inhomogeneous wareguide transformers. It is illustrated in this paper by examples of cascaded commensurate and noncommensurate transmission lines acting as impedance transformers for which the optima are known.
\end{abstract}

\section{INTRODUCTION}

M INIMAX response objectives, which can lead to equal-ripple optimum responses, are probably the most desirable objectives in microwave network

Manuscript received February 14, 1969; revised April 28, 1969. This work was carried out at the University of Manitoba with financial assistance from the Faculty of Graduate Studies of the University of Manitoba and from the National Research Council of Canada. This paper was presented at the 1969 IEEE G-MTT International Microwave Symposium, Dallas, Tex., May 5-7, 1969.

J. W. Bandler was with the Numerical Applications Group, Department of Electrical Engineering, University of Manitoba, Winnipeg, Canada. He is now with the Department of Electrical Engineering, McMaster University, Hamilton, Ont., Canada.

P. A. MacDonald is with the Numerical Applications Group, Department of Electrical Engineering, University of Manitoba, Winnipeg, Canada. optimization. This is because the designer is usually given an ideal response specification and has to synthesize a network which meets the ideal specification to within a specified maximum allowable deviation. For such problems a formulation which aims at reducing the maximum deviation of the response is the only one for which the optimum represents the best possible attempt at satisfying the design specifications within the constraints of the particular problem.

Methods for approaching minimax response optima and which can be used on networks whose parameters are constrained have been proposed [1]-[4]. The method described by Waren et al. [1], [2] reduces the constrained problem to a sequence of penalized unconstrained optimization problems, each one being started within the feasible region. The method of Ishizaki et al. [3], [4] reduces the original nonlinear problem to a series of linear programming problems. Assuming the methods converge, the minimax optimum can be arbitrarily closely approached.

If one raises the response deviation to a sufficiently high even power $p$ and uses that in the objective function, the maximum deviation can be reduced [4], [5]. The objective function becomes minimax as $p \rightarrow \infty$. Temes and Zai have recently described such a least $p$ th approximation method and its implementation [5].

In this paper a new optimization method called razor search is presented. The method, which is based on the pattern search technique of Hooke and Jeeves [6], was developed for the direct automatic optimization by computer of networks using as the objective function the maximum deviation of the response from the desired ideal response specification. Such a formulation will, in general, give rise to discontinuous partial derivatives of the objective function with respect to the network parameters [4], [7]-[11]. Under these circumstances otherwise efficient optimization methods-certainly on-line manual methods - may slow down or 
even fail to reach an optimum, particularly when the response hypersurface has a narrow curved valley along which the path of discontinuous derivatives lies [9]. This is probably the reason that success with the direct minimax formulation does not seem to have been previously demonstrated. To the authors' knowledge, the optimization of functions with discontinuous derivatives does not appear to have received much serious attention in the literature.

Essentially, the razor search strategy begins with a modified version of pattern search until this fails. A random point is selected automatically in the neighborhood (cf., Gelfand and Tsetlin [12]) and a second pattern search is initiated until this one fails. Using the two points where pattern search failed, a new pattern in the direction of the optimum is established and a pattern search strategy resumed until it too fails. This process is repeated until any of several possible terminating criteria is satisfied. Thus, the strategy should work on problems involving narrow "razor sharp" valleys in multidimensional space.

Since the only point of interest in the network response at any given time during optimization is that point where the maximum deviation occurs, it is important to obtain this point to any desired accuracy with as few response evaluations as possible. Another direct search method called ripple search, which locates the extrema of multimodal functions of one variable in an efficient manner, was developed for this purpose. Unlike the usual practice of sampling, for example, a frequency response at closely spaced fixed frequencies, the ripple search strategy first conducts a uniform search to determine the extrema and appropriate unimodal regions; subsequently, during optimization, it locates the extrema within the previously defined regions using a Fibonacci search scheme [13], [14]. Safeguards are built into the program to deal with continuously changing ripple patterns during optimization.

Descriptive and mathematical flow diagrams of the razor search and ripple search strategies are presented so that the methods can be readily programmed. Examples are also presented of the optimization of cascaded commensurate and noncommensurate transmission lines acting as impedance transformers for which the optima are known. Bandler describes the constrained optimization of inhomogeneous waveguide transformers using these methods in another paper [10]. In the present paper the advantages and disadvantages of the methods are discussed and ways of improving them are indicated.

\section{Problem Formulation}

Fig. 1 shows examples falling within the scope of this paper. In Fig. 1(a) the problem is to maximize the minimum gain of an amplifier over a frequency band of interest subject to a maximum allowable gain. In Fig. 1(b) the problem is to minimize the maximum deviation of the gain from a desired gain. In Fig. 1(c) the problem is to minimize the maximum reflection coefficient of a matching network. A wide range of microwave network design problems can be formulated along these or similar lines. Note that the response specification need not be linear.

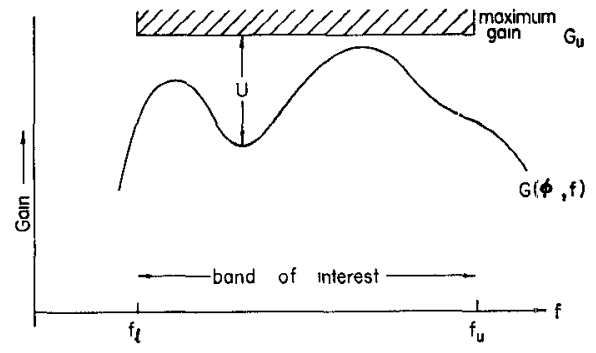

(a)

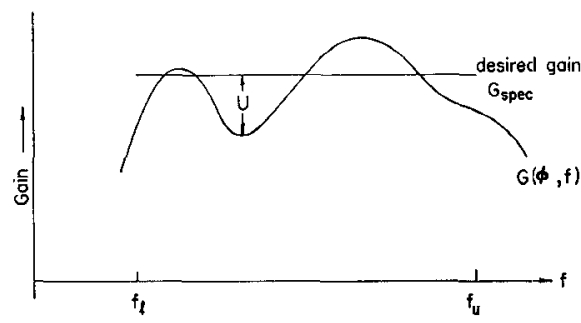

(b)

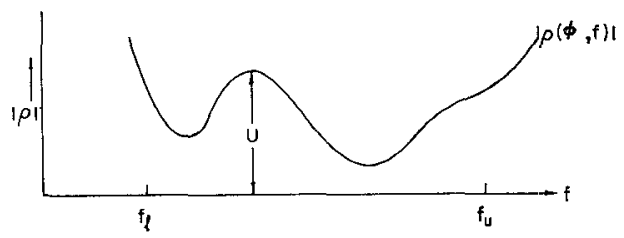

(c)

Fig. 1. Examples having minimax response objectives and falling within the scope of this paper.

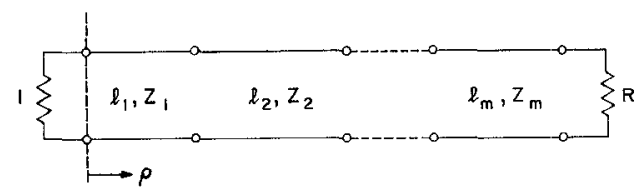

Fig. 2. $m$-section resistively terminated cascade of transmission lines.

A detailed discussion on the formulation of direct minimax response objectives in general is presented elsewhere in this issue [11]. Also discussed in that paper are methods of dealing with parameter and other constraints subject to which the optimization is to be carried out (see also Bandler [10]). The present paper is, therefore, devoted to a presentation of the razor search and ripple search strategies and an examination of their behavior on essentially unconstrained test problems.

The test problems are examples of the optimization of cascaded commensurate and noncommensurate transmission lines acting as impedance transformers between resistive terminations as shown in Fig. 2. A previous numerical investigation [8] found that the optimum designs were, not unexpectedly, quarter-wave Chebyshev transformers [15]. So the results obtained by optimization may be compared with the results obtained by analytic methods. It should be born in mind, however, that none of the well-known properties of quarter-wave transformers are employed to simplify the process-as far as the optimization strategies are concerned the problems are quite arbitrary. 


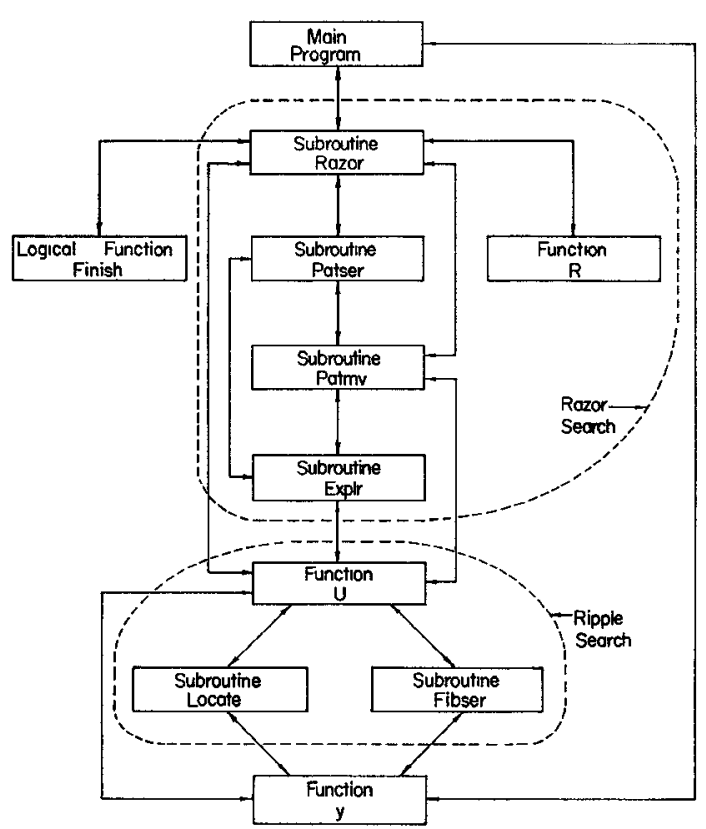

Fig. 3. Block diagram summarizing the computer program structure and indicating the relative hierarchy of the subprograms.

The objective for a matching network [Fig. 1(c)] is to find

$$
\check{U}=\min _{\boldsymbol{\phi}}(U)=\min _{\boldsymbol{\phi}}\left\{\max _{\left[f_{l}, f_{u}\right]}[|\rho(\boldsymbol{\phi}, f)|]\right\}
$$

where $\phi$ represents the variable parameters of the network, $\rho$ is the reflection coefficient, $f$ is frequency, and $f_{l}$ and $f_{u}$ are the lower and upper band edges, respectively. $U$ is seen to be the maximum magnitude of $\rho$ in the band, and the objective is to find a set of parameter values $\check{\boldsymbol{\phi}}$ which minimizes $U$ (expressions of the form of (1) can be written down for the other examples in Fig. 1).

A computer program was written to calculate the reflection coefficient of a resistively terminated cascade of transmission lines at any frequency and for any section lengths and characteristic impedances as shown in the Appendix. It is in the form of a function subprogram to be called by the ripple search package (Section IV).

\section{The Razor Search Strategy}

The razor search strategy presented in this paper employs a pattern search strategy which is different from published versions of pattern search [6], [16]-[18] in the following ways:

1) The exploratory increments depend on the total progress made between the previous two base points. Thus, they automatically increase or decrease in accordance with previous successes or failures, respectively.

2) When a pattern move plus exploratory moves fail the pattern is not immediately discarded. Instead, the same procedure is repeated closer to the base point. If this too is unsuccessful, the procedure is attempted in the opposite direction.

Fig. 3 shows a block diagram summarizing the computer program. The razor search strategy is outlined by the descriptive flow diagrams of Figs. 4 to 7. Also shown in these

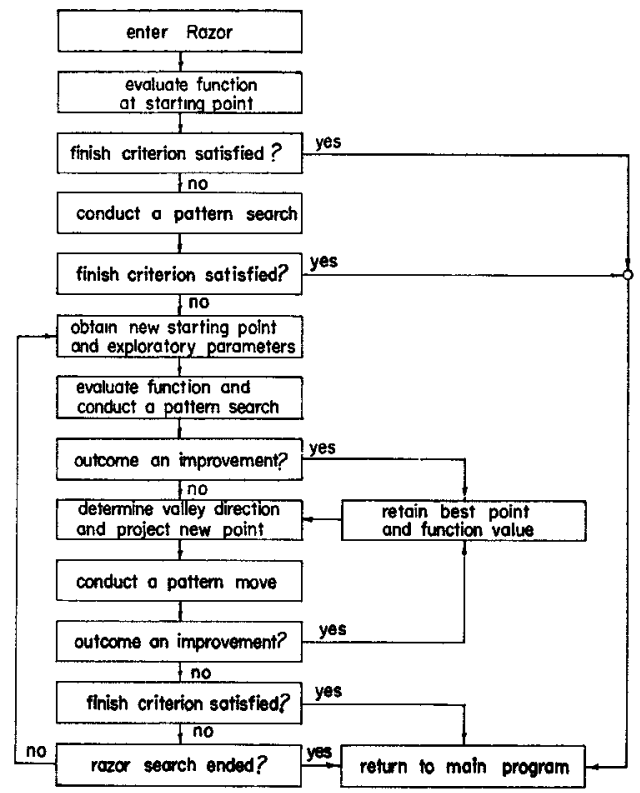

(a)

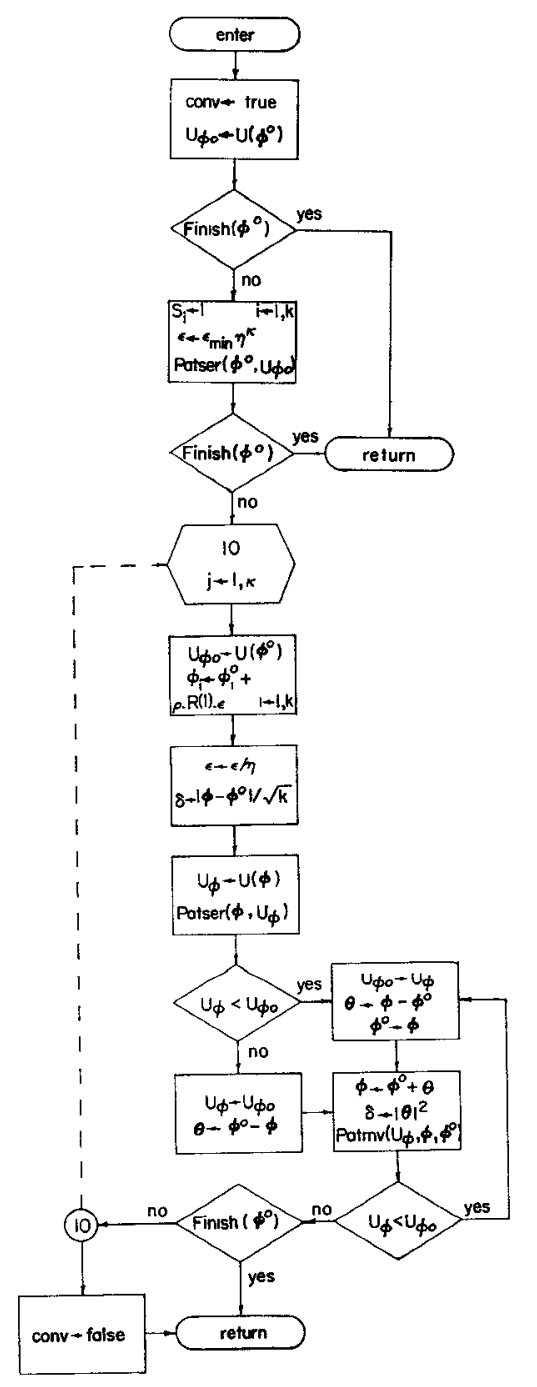

(b)

Fig. 4. (a) Descriptive flow diagram of subroutine RAzOR. (b) Mathematical flow diagram of subroutine RAZOR. 


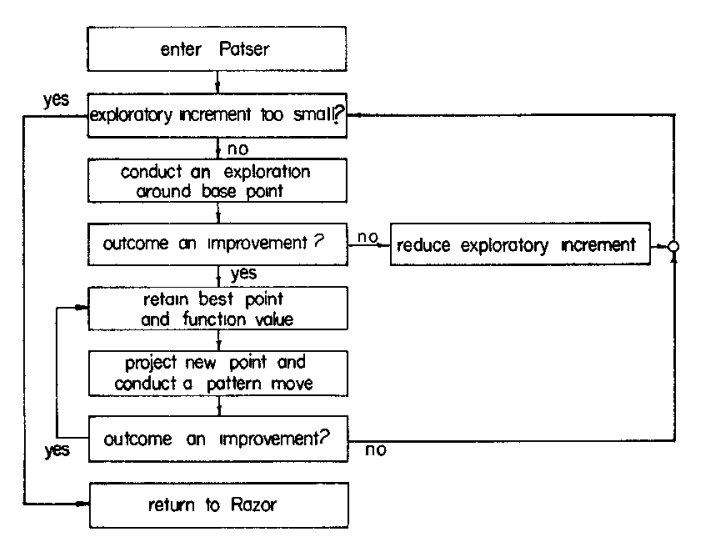

(a)

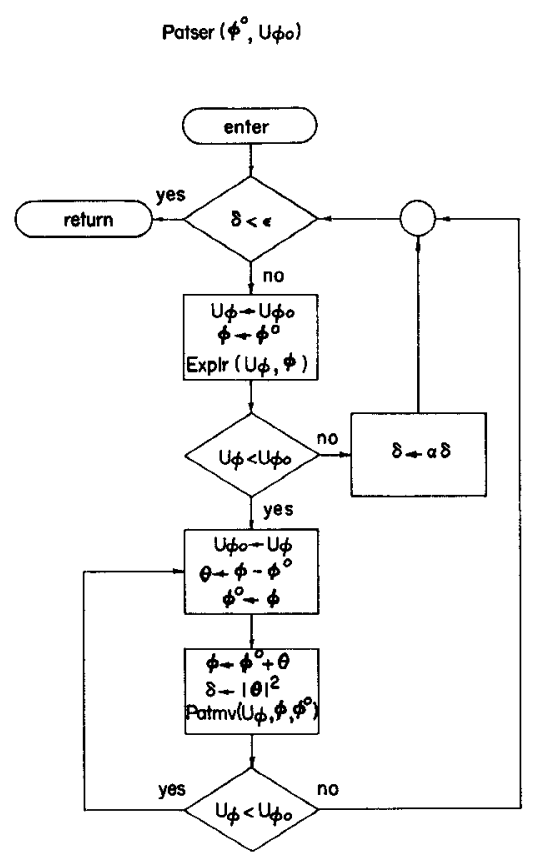

(b)

Fig. 5. (a) Descriptive flow diagram of subroutine PATSER. (b) Mathematical flow diagram of subroutine PATSER.

figures are the essential details of the computer program in symbolic form. ${ }^{1}$ Perhaps the best way of comprehending the strategy is by studying an example which demonstrates most of the main features, and by discussing their general implications.

The problem of optimizing a 2-section transmission-line transformer for a load to source impedance ratio of $10: 1$ over a 100 percent bandwidth with the section lengths fixed at their optimum values, i.e., quarter-wave at center frequency, is a good choice because ordinary pattern search fails to reach the optimum under certain circumstances [9].

\footnotetext{
I Details such as counters for the numbers of function evaluations made in the subroutines, and other statements not directly involved in the logic of the optimization process, are not shown.
}

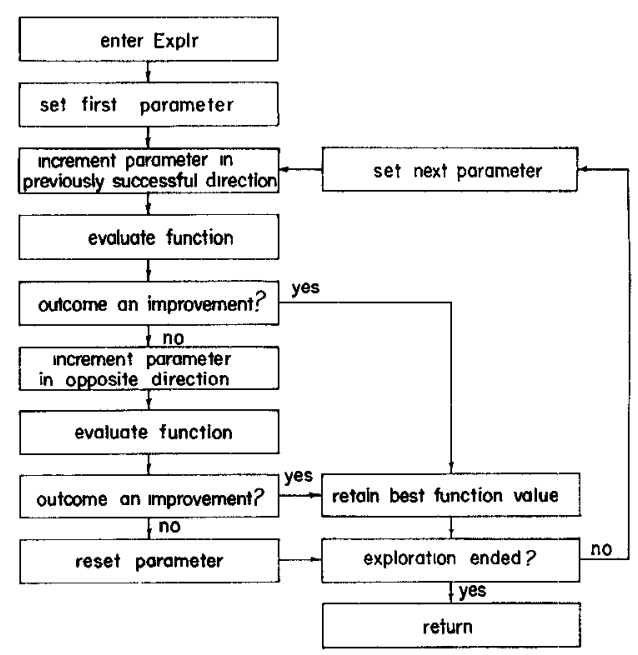

(a)

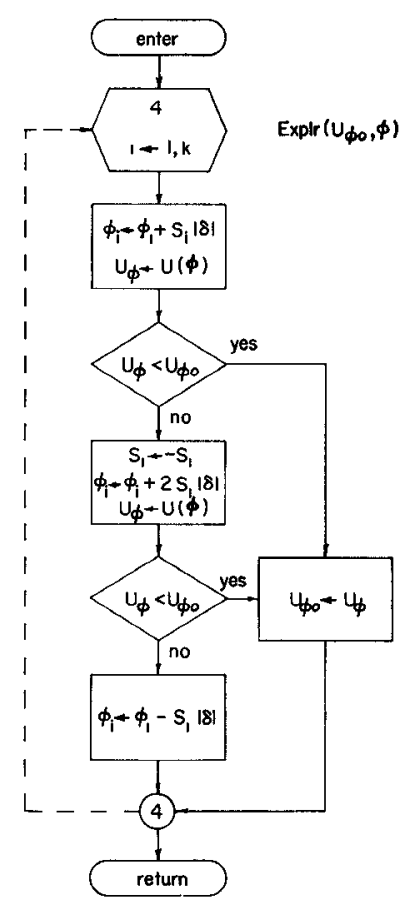

(b)

Fig. 6. (a) Descriptive flow diagram of subroutine EXPLR. (b) Mathematical flow diagram of subroutine EXPLR.

Formally, the problem is to reach

$$
\check{U}=\min _{\boldsymbol{\phi}}(U)=\min _{\boldsymbol{\phi}}\left\{\max _{[0.5,1.5]}[|\rho(\boldsymbol{\phi}, f)|]\right\}
$$

where the center frequency is taken as $1 \mathrm{GHz}$. It is convenient to define

$$
\phi \equiv\left(\phi_{1}, \phi_{2}\right) \equiv\left(Z_{1}, Z_{2}\right) .
$$

Contours of $U$ versus $Z_{1}$ and $Z_{2}$, the characteristic impedances of the two sections normalized to the source impedance, are plotted in Fig. 8. The sharp points in the contours indicate the presence of the discontinuous derivatives which arise when $U$ jumps from one response ripple extremum to another. 


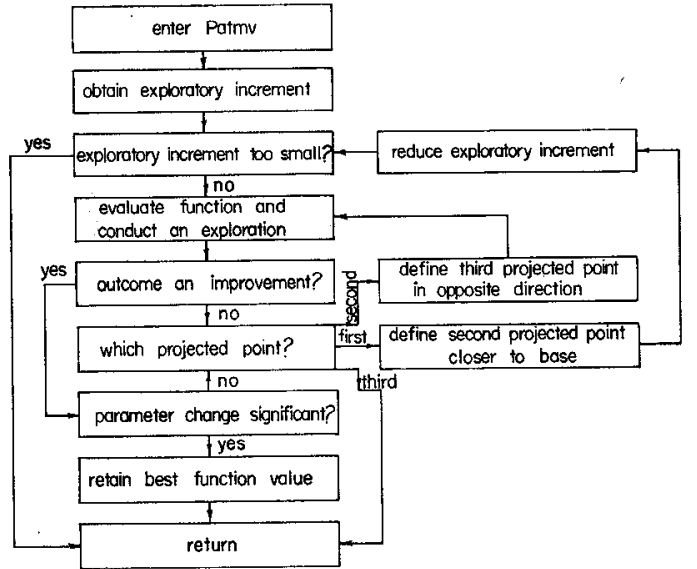

(a)

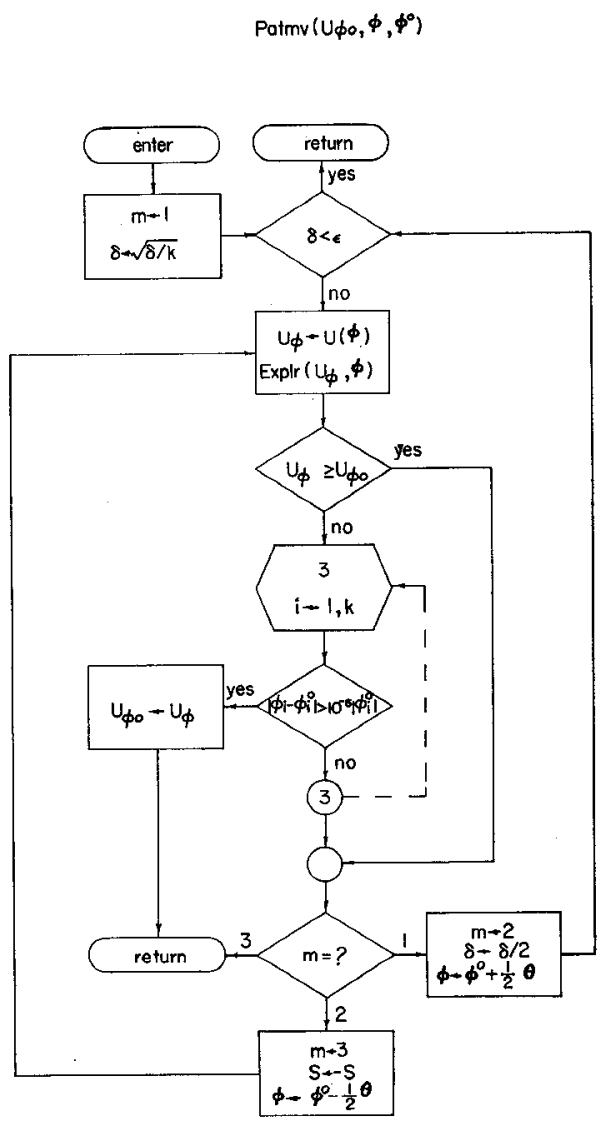

(b)

Fig. 7. (a) Descriptive flow diagram of subroutine PATMV. (b) Mathematical flow diagram of subroutine PATMY.

The notation used in Figs. 4 to 7 is defined in the Appendix. For the purpose of the present discussion superscripts will denote sequential parameter or function values obtained during optimization. It is important to note that in Figs. 4 to $7, \phi$ refers to any current projected, exploratory or random point, while $\phi^{\circ}$ is temporarily the best point. Thus, a particular set of parameter values may be differently defined, depending upon which subroutine it finds itself in.

The starting point $\phi^{1}=(1.25,4.50)$ is selected as the first base point $\phi^{\circ}$ on entering RAZOR (see Fig. 4). The objective function is evaluated at $\phi^{1}$. Let its value $U_{\phi 0}$ be denoted $U^{1}$. A

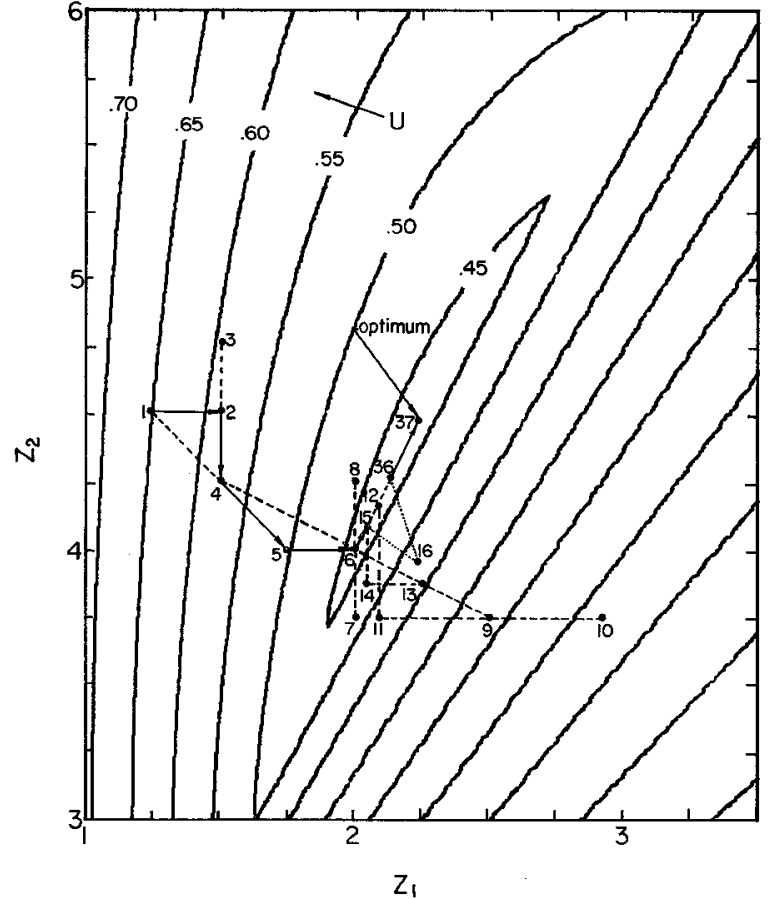

Fig. 8. Example illustrating the razor search strategy showing how following one random move the path of discontinuous derivatives leading to the optimum is effectively located.

user supplied finish criterion is tested next. ${ }^{2}$ If this is not satisfied we prepare for a pattern search. The vector $S$, which keeps track of previous successful directions for the exploratory moves, has all its components initially set at 1 . It is also seen that

$$
\epsilon \leftarrow \epsilon_{\min } \eta^{\kappa}
$$

where $\epsilon$ is the current minimum permissible exploratory increment, $\kappa$ is the maximum number of random moves to be allowed, and $\eta$ is a scale factor (greater than or equal to unity); $\epsilon_{\min }$ is the minimum possible value of $\epsilon$. Subsequently, every time a random move is made

$$
\epsilon \leftarrow \epsilon / \eta
$$

This feature is included to prevent the parameter increments from becoming too small during the early stages of optimization which would probably result in wasted effort. In the present example $\epsilon_{\min }=0.01, \kappa=3$, and $\eta=2$; therefore initially $\epsilon=0.08$.

Before attempting a pattern search (see Fig. 5) the exploratory increment $\Delta \phi(=\delta$ in the program at this stage $)$ is compared with $\epsilon$. Initially, in this example, $\Delta \phi=0.25>\epsilon$ $=0.08$. Exploration begins with $\phi_{1}$ as indicated in Fig. 6 and takes us to $\phi^{2}$. Since $U^{2}<U^{1}$ we retain $\phi^{2}$ and continue exploration with $\phi_{2}$. The next point $\phi^{3}$ is rejected because $U^{3}>U^{2}$ and $\phi_{2}$ is incremented in the opposite direction to

${ }^{2}$ The finish criterion can take several forms. For example, a test could be made to determine whether the maximum deviation has fallen below some specified value. Alternatively, a test could be made to determine whether any significant improvement has occurred since the last one or more times the finish criterion was tested. One could even check the number of response extrema and use it as a finish criterion. 
$\boldsymbol{\phi}^{4} \cdot U^{4}<U^{2}$ so $\boldsymbol{\phi}^{4}$ is retained in place of $\boldsymbol{\phi}^{2}$. The first set of exploratory moves is now complete. Returning to PATSER (Fig. 5) we find that $U^{4}<U^{1}$.

The point $\phi^{4}$ becomes the second base point $\dot{\phi}^{0}$ and in accordance with the pattern move strategy we obtain a projected point $\boldsymbol{\phi}^{5}$ such that $\boldsymbol{\phi}^{5}-\boldsymbol{\phi}^{4}=\boldsymbol{\phi}^{4}-\boldsymbol{\phi}^{1}$, i.e., in general

$$
\phi \leftarrow \phi^{\circ}+\theta
$$

where $\theta$ is the vector difference between the last two base points. Before proceeding with the pattern move strategy of Fig. 7 the exploratory increment is compared with $\epsilon$. The value of $\Delta \phi$ is $\left|\phi^{4}-\phi^{1}\right| / \sqrt{ } k$, where $k$ is the dimensionality of the space; here $k=2$. Each parameter was successfully incremented during the previous exploration; therefore, $\Delta \phi$ remains at 0.25 (otherwise the increment would have been automatically reduced). Since the exploratory increment is not too small, $U^{5}$ is evaluated, and the next set of exploratory moves is started. Incrementing $\phi_{1}$ in the direction previously found successful takes us to $\phi^{6}$. It is found that $U^{6}<U^{5}$ so we retain $\phi^{6}$ and increment $\phi_{2}$ in the direction previously found successful for this parameter. Thus, $\phi_{2}$ is first decreased because $S_{2}=-1$. However, neither $\phi^{7}$ nor subsequently $\boldsymbol{\phi}^{8}$ result in any improvement over $\dot{\phi}^{6}$.

The outcome of the pattern move plus exploration is an improvement because $U^{6}<U^{4}$ and the parameter change is also significant (see Fig. 7). Thus, $\phi^{6}$ becomes the third base point. We now obtain a projected point $\boldsymbol{\phi}^{9}$ such that $\phi^{9}-\dot{\phi}^{6}$ $=\phi^{6}-\dot{\phi}^{4}$ and an exploratory increment $\Delta \phi=\left|\phi^{6}-\phi^{4}\right| / \sqrt{ } k$ $=\sqrt{ } 10 / 8$. Exploration around $\phi^{9}$ ends at $\phi^{12}$ with $U^{12}<U^{9}$. It is unsuccessful, however, since $U^{12}>U^{6}$.

Not wishing to destroy the pattern already established, we project a point midway between $\phi^{6}$ and $\phi^{9}$ to $\phi^{13}$ and reduce the exploratory increment appropriately. This is shown in Fig. 7, where it is also seen that $m$, the counter for projected points, is set at 2 . We finally arrive at $\phi^{15}$ which is an improvement over $\dot{\phi}^{6}$. However, on entering PATMV it is found that $\Delta \phi<\epsilon$. The first pattern search is, therefore, terminated at $\phi^{15}$.

Returning to RAZOR the finish criterion is tested. If this is not satisfied we prepare for the first random move. The random point $\dot{\phi}^{16}$ is given by the instruction

$$
\phi_{i} \leftarrow \phi_{i}{ }^{\circ}+\rho \cdot R(1) \cdot \epsilon \quad i=1,2, \cdots, k
$$

where $\rho$ (not to be confused with reflection coefficient) is a scale factor and $R(1)$ generates random numbers between -1 and 1 . The minimum exploratory increment is reduced in accordance with (5) and $\delta=\Delta \phi=\left|\phi^{16}-\phi^{15}\right| / \sqrt{ } k$.

A second pattern search is conducted starting at $\phi^{16}$. Eventually we arrive at $\boldsymbol{\phi}^{36}$ where this pattern search is abandoned because $\Delta \phi<\epsilon=0.04$. The values $U^{36}$ and $U^{15}$ are compared. Since $U^{36}<U^{15}$ the direction of the valley indicated in Fig. 8 is given by $\dot{\phi}^{36}-\dot{\phi}^{15}$. Taking $\boldsymbol{\phi}^{36}$ as a base point and $\dot{\phi}^{37}$ as a projected point down the valley such that $\phi^{37}-\phi^{36}=\phi^{36}-\phi^{15}$, we continue with the pattern move strategy until $\Delta \phi<0.04$. Then the finish criterion is tested. If this is not satisfied we prepare for the next random move.

Table I summarizes the important steps in this example. Note that $\phi^{37}=(2.21791,4.44943)$. Thus, at the 37 th func-
TABLE I

Summary of the Important Steps In the EXAMPLE Illustrating the Razor Search Strategy

\begin{tabular}{|c|c|c|}
\hline Point & Description & Value \\
\hline 1 & Initial base point $\boldsymbol{\phi}^{1}$ & $\phi^{1}=(1.25,4.5)$ \\
\hline $2,3,4$ & $\begin{array}{l}\text { Exploratory points } \phi^{2}, \phi^{3}, \phi^{4} \\
\quad \text { from } \phi^{1} \text {. } \\
\text { Second base point is } \phi^{4} \text {. }\end{array}$ & $\phi^{4}=(1.5,4.25)$ \\
\hline 5 & Projected point $\phi^{5}$. & \\
\hline $6,7,8$ & $\begin{array}{l}\text { Exploratory points } \phi^{6}, \phi^{7}, \phi^{8} \\
\text { from } \phi^{5} \text {. } \\
\text { Third base point is } \phi^{6} \text {. }\end{array}$ & $\phi^{6}=(2.0,4.0)$ \\
\hline 9 & Projected point $\phi^{9}$ & \\
\hline $10,11,12$ & $\begin{array}{l}\text { Exploratory points } \phi^{10}, \phi^{11} \\
\phi^{12} \text { from } \phi^{9} \text {. }\end{array}$ & \\
\hline & Projected point $\boldsymbol{\phi}^{13}$ & \\
\hline 14,15 & $\begin{array}{l}\text { Exploratory points } \phi^{14}, \phi^{15} \\
\text { from } \phi^{13} \text {. } \\
\text { First pattern search termi- } \\
\text { nates at } \phi^{15} \text {. }\end{array}$ & $\phi^{15}=(2.05236,4.07264)$ \\
\hline 16 & $\begin{array}{l}\text { Random point } \phi^{16} \text { projected } \\
\text { from } \phi^{15} \text {. }\end{array}$ & $\phi^{16}=(2.23389,3.95552)$ \\
\hline 36 & $\begin{array}{l}\text { Second pattern search termi- } \\
\text { nates at } \phi^{36} \text {. }\end{array}$ & $\phi^{36}=(2.13514,4.26104)$ \\
\hline 37 & $\begin{array}{l}\text { Point } \phi^{37} \text { projected down } \\
\text { valley using } \phi^{15} \text { and } \phi^{36} \text {. }\end{array}$ & $\boldsymbol{\phi}^{37}=(2.21791,4.44943)$ \\
\hline
\end{tabular}
$U^{1}=0.64907 \quad U^{37}=0.42986 \quad \check{U}=0.42857$

tion evaluation the parameters are within about 1 percent and $\frac{1}{2}$ percent, respectively, of their values at the optimum $\breve{\phi}=(2.2361,4.4721)$.

\section{The Ripple Search Strategy}

The program for ripple search is in three parts. First, there is the function subprogram $U$ which is represented by the flow diagram of Fig. 9. Secondly, there is the subroutine LOCATE represented in Fig. 10. Thirdly, there is a subroutine called FIBSER which conducts the Fibonacci search [14] for an extremum. FIBSER is not shown as it is a version of a published algorithm [13] which has been slightly modified to handle both maxima and minima. The notation used in Figs. 9 and 10 is defined in the Appendix.

Refer to Fig. 9. When $U$ is called for the first time by RAZOR $j=j^{\prime}=0$. As a consequence it is seen that LOCATE is immediately called. As shown in Fig. 10, LOCATE sets $o_{1}$, the location of the first extremum, and $e_{1}$, the left hand end point of the first unimodal interval (see Fig. 11). A test is then made to determine whether the first turning point (if any) will be a local minimum as in Fig. 11 or a local maximum. Following this a uniform search loop is entered. This keeps a record of $y_{t}$, the temporary maximum value of the objective function $y$. The locations of the local extrema are recorded sequentially as $o_{1}, o_{2}, o_{3}, o_{4}, \cdots$ as indicated in Fig. 11. End points of intervals subsequently to be explored by FIBSER are first defined here. Denoted $e$ they are located midway between adjacent extrema with the exception of the first and last as shown in Fig. 11. Finally, the maximum value of $y$ obtained is set equal to $U$.

This process, i.e., the search for $U$ by uniformly spaced test points, will be repeated next time $U$ is called until $j$ changes to 1 . Since $j$ in RAZOR is not set equal to 1 until the 


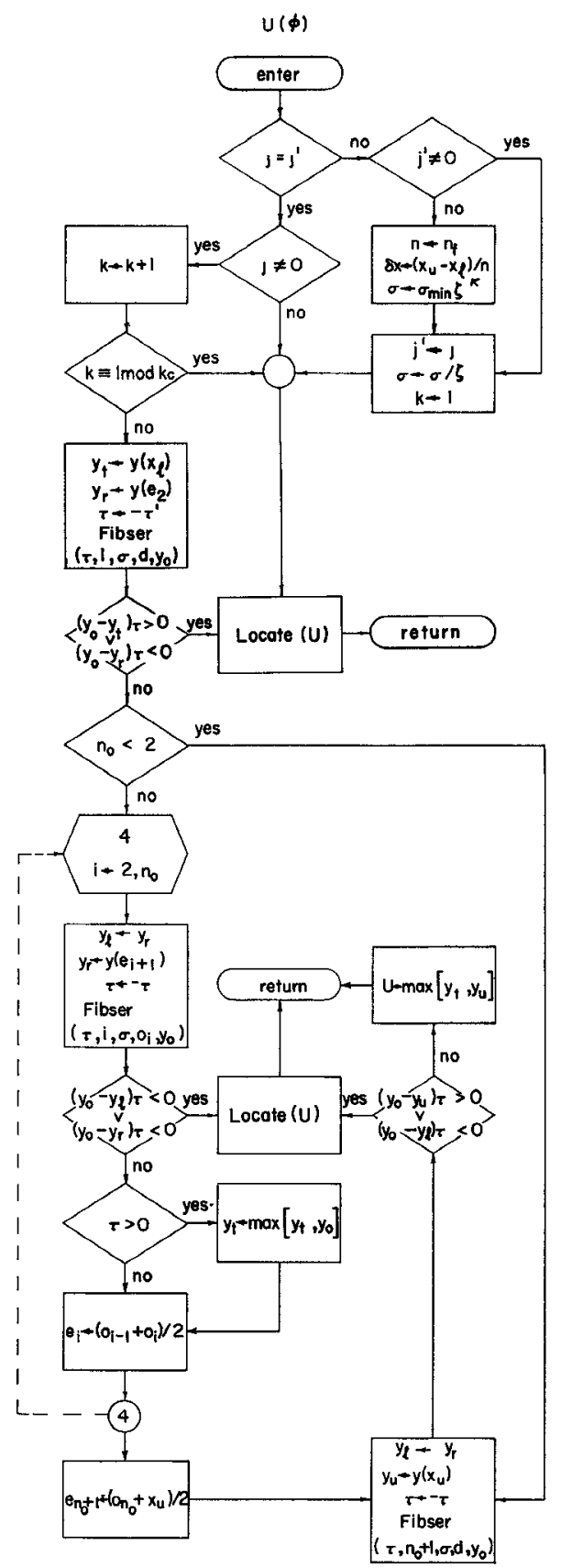

Fig. 9. Flow diagram of function $U$.

first random move is made, this means that during the first pattern search the response is uniformly sampled. When $j=1, j^{\prime}$ is still 0 so, as shown by Fig. 9 , a new increment (preferably smaller than before) is set for any subsequent uniform searches.

Also,

$$
\sigma \leftarrow \sigma_{\min } \zeta^{\kappa}
$$

where $\sigma$ is the uncertainty interval in the Fibonacci search; $\kappa$ is the maximum number of random moves in RAzOR and $\zeta$ is a scale factor (greater than or equal to unity); $\sigma_{\min }$ is the minimum value of $\sigma$. Accompanying every random move in RAZOR, therefore,

$$
\sigma \leftarrow \sigma / \zeta
$$

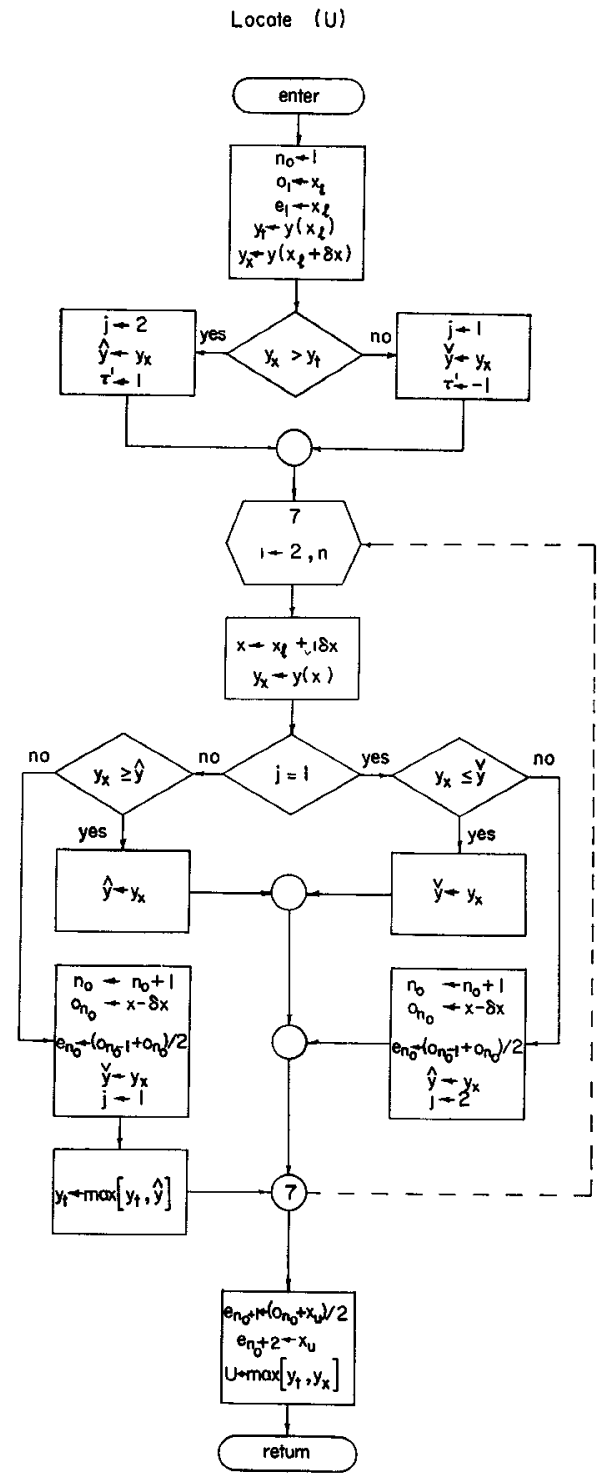

Fig. 10. Flow diagram of subroutine LOCATE.

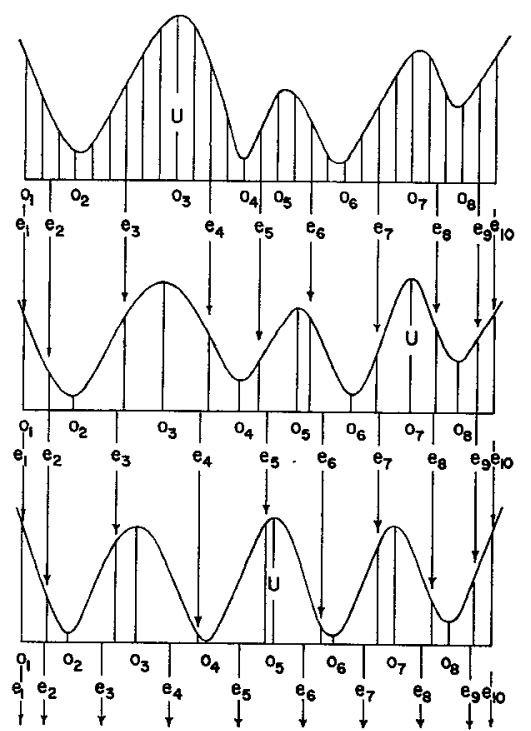

Fig. 11. Example illustrating the ripple search strategy showing how a uniform search defines end points of intervals subsequently to be explored by a series of Fibonacci searches. 
This feature is similar to the one in the razor search strategy which prevents the parameter increments from becoming too small. In the present case we do not want the uncertainty interval to be too small in the early stages of optimization, which would be wasteful. $j^{\prime}$ is now set equal to $j$ and the counter $k$ to 1 . A final uniform search is then made before FIBSER is called for the first time.

Next time $\mathrm{U}$ is called (Fig. 9), $j=j^{\prime}=1$, so unless $k \equiv 1$ $\bmod k_{c}$ (in which case a uniform search is carried out) a series of Fibonacci searches is made to locate the extrema within the previously defined intervals to intervals of uncertainty $\sigma$. If the first argument in the call statement of FIBSER, namely $\tau$, is +1 a maximum is expected, if it is -1 a minimum is expected. Notice that following each Fibonacci search certain safety checks are made. These determine whether the response is consistently divided up into a monotonically increasing or decreasing portion followed appropriately by a series of maxima and minima and ending in a monotonic portion. These tests are important because the series of end points, which are continually being redefined (as indicated in Fig. 11), were set after a previous search and may not correspond to the present ripple pattern. For example, the ripples may have shifted substantially or a new one may have appeared and so on. Thus, if any such inconsistency is detected, LOCATE is called so that the search for $U$ becomes uniform and the end points are redefined in accordance with the current ripple pattern. If no inconsistency occurs the end points are redefined in $U$ using the current local extrema, and the maximum value of $y$ obtained is set equal to $U$.

\section{NuMERICAL RESULTS}

Table II shows typical results obtained for the 2-section example defined by (2) and (3) starting from each corner of Fig. 8. Two runs per corner were made, one with an initial exploratory increment of 0.25 , the other with an increment of 0.5. Data not shown in Table II but relevant to the strategy is

$$
\begin{aligned}
\alpha & =0.1 \\
\epsilon_{\min } & =10^{-5} \\
\eta & =5 \\
\kappa & =5 \\
n & =8 \\
n_{f} & =100 \\
k_{c} & =2000 \\
\sigma_{\min } & =10^{-3} \\
\zeta & =2 .
\end{aligned}
$$

The number of function evaluations required to bring the maximum reflection coefficient to within 0.01 percent of its optimum value before the next random move was made is shown for each case. The maxima in each response agree to at least five significant figures (but this is not in itself an indication that the optimum has been reached). Fig. 12 shows responses corresponding to the four starting points and the optimum response.
TABLE II

Optimization of a 2-Section 10:1 Quarter-Wave Transformer OVER 100 PERCENT BANDWIDTH

\begin{tabular}{cc|c|c|c|c}
\hline \multicolumn{2}{c|}{ Starting Point } & & & $\begin{array}{c}\text { Number } \\
\text { of ran- } \\
\text { don } \\
\text { moves }\end{array}$ & $\begin{array}{c}\text { Number } \\
\text { of function } \\
\text { eval- } \\
\text { uations }\end{array}$ \\
\hline$Z_{1}$ & $Z_{2}$ & $\delta$ & $\rho$ & 2 & 157 \\
\hline 1.0 & 3.0 & 0.25 & 10 & 2 & 207 \\
1.0 & 3.0 & 0.5 & 10 & 2 & 34 \\
1.0 & 6.0 & 0.25 & - & 0 & 152 \\
1.0 & 6.0 & 0.5 & 10 & 2 & 223 \\
3.5 & 6.0 & 0.25 & 5 & 2 & 100 \\
3.5 & 6.0 & 0.5 & 5 & 1 & 210 \\
3.5 & 3.0 & 0.25 & 5 & 3 & 163 \\
3.5 & 3.0 & 0.5 & 10 & 1 & \\
\hline
\end{tabular}

The number of random moves and function evaluations required to bring the reflection coefficient within 0.01 percent of its optimum value are shown.

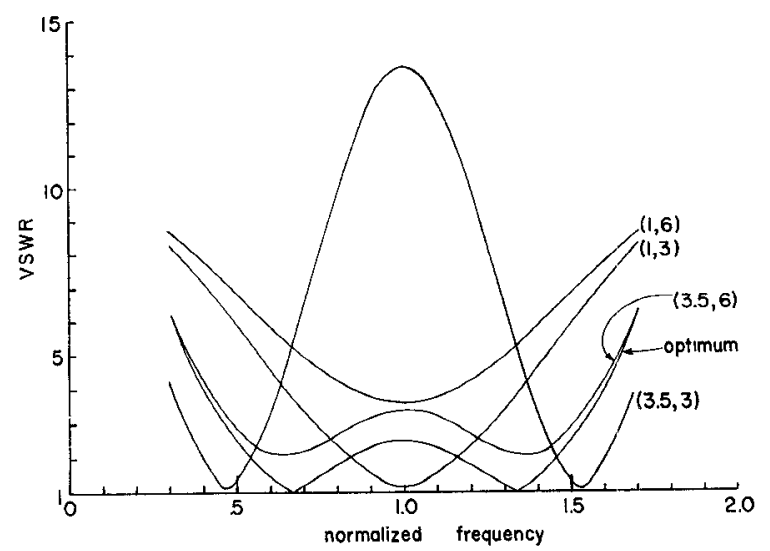

Fig. 12. Responses of the 2-section transformer corresponding to the starting points tabulated in Table II. The optimum response is also shown.

Extensive experimentation with the various constants and scale factors to reduce the number of function evaluations has not yet been attempted so these results should not be regarded as the best possible. A published version of pattern search [16], [17] on the other hand performed rather poorly on this problem. It terminated outside the bounded area starting from $(1,3)$, and only came reasonably close to the optimum starting from other corners when the initial increment was 0.25 . The reader is referred to another publication [9] for more details on the behavior of pattern search.

Table III presents some results obtained for 3-section 10:1 transformers optimized over 100 percent bandwidth. The relevant data for the three examples is the same as for the previous examples with the following exceptions:

$$
\begin{aligned}
\delta & =0.25 \\
\rho & =5 \\
n & =12 .
\end{aligned}
$$

The starting points for the first two cases essentially represent optimum single-section quarter-wave transformers (see curve $a$ in Fig. 13). In the first one the section lengths are held fixed at the optimum quarter-wave value $l_{q}$. In the second one they are allowed to vary. The third case can be 
TABLE III

Optimization of a 3-Section 10:1 Transformer over a 100 Percent Bandwidth

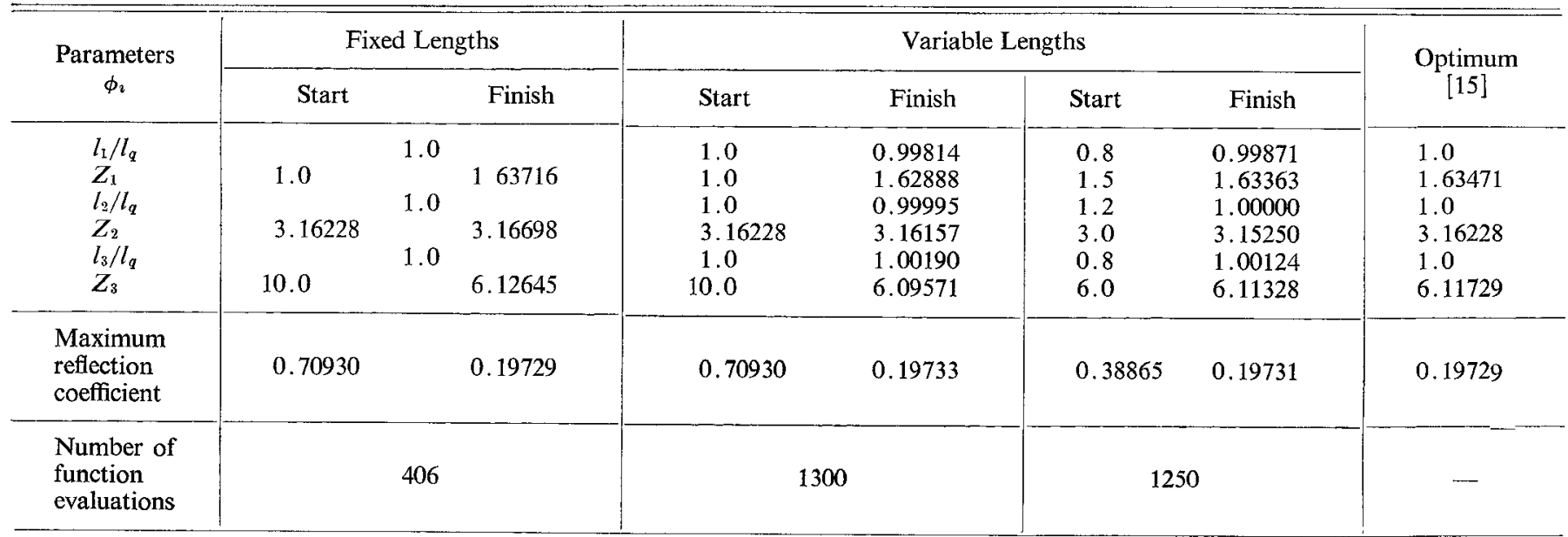

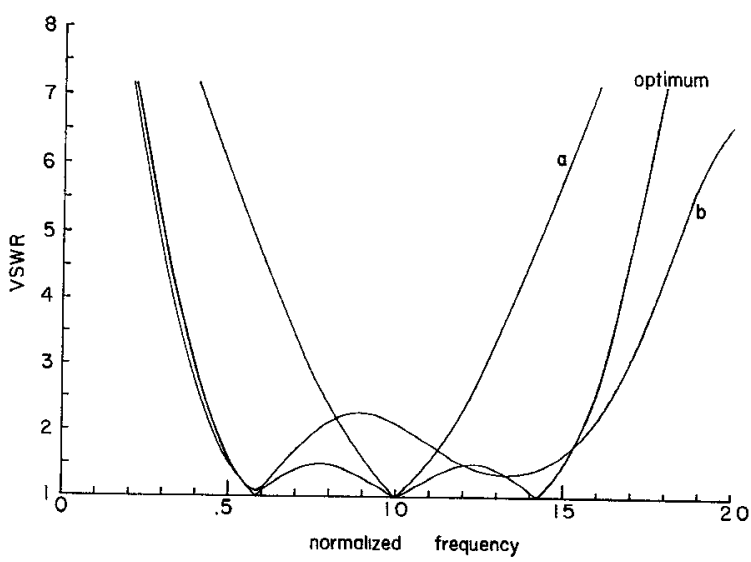

Fig. 13. Responses of the 3-section transformer corresponding to the starting points tabulated in Table III. Curve $a$ corresponds to the first two cases, curve $b$ to the third case. The optimum response is also shown.

thought of as a reasonable guess at an optimum 3-section transformer (see curve $b$ in Fig. 13), and both lengths and impedances are allowed to vary.

The number of function evaluations and the maximum reflection coefficient before and after optimization are shown for each case in Table III. Optimization was continued until all 5 random moves had been made and the exploratory increments had fallen below $10^{-5}$. Again all maxima in each response agreed to 5 significant figures. The optimum response is shown in Fig. 13. Observe that neither of the initial responses has as many extrema as the optimum response. Furthermore, as the transformer corresponding to curve $a$ has essentially one section, one could, in a rather loose way, say that two additional sections have "grown" during optimization.

It should be emphasized that the small differences between the results obtained by optimization and the analytic results [15] are not attributable to any a priori approximations in the formulation. These differences, which do, however, reflect the efficiency of the optimization process as a function of the input data determining the strategy, can be reduced simply by continuing the optimization process.

\section{DISCUSSION}

The razor search strategy was tested on Rosenbrock's function [19], [20]

$$
U=100\left(\phi_{2}-\phi_{1}^{2}\right)^{2}+\left(1-\phi_{1}\right)^{2}
$$

in order to make an adequate comparison with other direct search methods on a problem not involving discontinuous derivatives and to assess the effects of the modifications described in Section III. A contour diagram of (10) is given by Bandler [11].

Table IV presents the comparison. Typical results obtained with razor search with and without random moves are shown. Without random moves razor search compares very favorably with previous versions of pattern search, with random moves it compares favorably with Rosenbrock's method. It may be conjectured, therefore, that a random move is ultimately more efficient at locating an improved direction of search along a narrow valley than is reducing the exploratory increments. When the path of discontinuous derivatives lies along such a valley as in Fig. 8 , where exploring parallel to the coordinate axes from this path yields no improvement, a random move is obviously more efficient.

Powell's method [21], [22] which performs very well on Rosenbrock's function (since it has quadratic convergence) was also tried on the example defined by (2) but failed in much the same way as pattern search after one linear minimization.

O'Hagan's spider search method [23], also based on pattern search, but which explores in randomly chosen orthogonal directions, should ultimately be successful because of the finite probability of obtaining a direction yielding improvement [24]. This suggests that a rotation of coordinates coupled with the facility of a random move could result in greater efficiency. Another possibility is to use three base points to make a pseudo-quadratic extrapolation so as to get a better estimate of the path of discontinuous derivatives. Following this, a one-dimensional minimization could be made to find the minimum along that path. 
TABLE IV

A Comparison of Razor Search with Published Results of Some Other Direct Search Methods on Rosenbrock's Function

\begin{tabular}{l|c|c}
\hline \multicolumn{1}{c|}{ Strategy } & $U$ & $\begin{array}{c}\text { Function } \\
\text { Eval- } \\
\text { uations }\end{array}$ \\
\hline Ordinary pattern search [19] & $8.03 \times 10^{-1}$ & 200 \\
Pattern with adjusted steps [19] & $1.03 \times 10^{-2}$ & 200 \\
Razor search without random moves & $7.4 \times 10^{-3}$ & 200 \\
Rotating coordinates [20] & $2.2 \times 10^{-5}$ & 200 \\
Razor search with 3 random moves & $1.6 \times 10^{-6}$ & 172 \\
Powell's method [21] & $7 \times 10^{-10}$ & 151 \\
Minimum & 0 & - \\
\hline
\end{tabular}

The ripple search method might be made more efficient if quadratic interpolation [11] is employed to find the extrema, rather than the Fibonacci search. But in either case there is the danger that if the maxima are not accurately evaluated it may be difficult for razor search to determine a direction of improvement when any two maxima are nearly equal. The additional function evaluation made just prior to every random move (Fig. 4) when the uncertainty interval for the Fibonacci search is reduced is included to alleviate this difficulty to some extent.

To date, razor search and ripple search have been successfully applied to optimization problems having up to 14 variables and 8 ripple maxima. More experience is required, however, before the practical limitations of the methods are realized. A worthwhile investigation would be an in-depth comparison of the methods with the other currently available methods for approaching minimax optima [1]-[5]. It is computation time, reliability in a wide range of problems and ease of implementation that should form the basis of any comparison.

\section{CONCLUSIONS}

Efficient direct search methods have been presented for the computer-aided optimization of networks for which minimax response optima are desired. In this paper the methods have been tested on problems for which the optima are known. In another paper [10] these methods are applied to the constrained optimization of inhomogeneous waveguide transformers (including parasitic junction discontinuity effects).

The methods described should find immediate application to a wide range of microwave network design problems, particularly where optimum broadband performance is required. Noncommensurate network components present no special difficulties. Parasitic effects can be taken into account if reliable data to represent them is available. Response and parameter constraints can also be taken into account to guarantee physical realizability. In common with other optimization methods, generally the closer the starting point is to a local optimum the faster will be the convergence onto that optimum. So, if an approximate feasible solution can be found by "exact" methods, this can be used as a starting point.

\section{APPENDIX}

The Razor Search Package

Variables in RAZOR as shown in Fig. 4:

conv initially true, becomes false unless finish criterion satisfied

$j$ counts number of random moves

$k$ dimensionality of space

$R(1)$ generates random numbers between -1 and 1

$S$ vector controlling directions for exploratory moves

$S_{i} \quad$ ith component of $S$

$U$ objective function to be minimized

$U_{\phi}$ value of $U$ at $\phi$

$U_{\phi \mathrm{o}} \quad$ value of $U$ at $\phi^{\circ}$

$\delta$ defines next exploratory increment

$\epsilon$ current minimum permissible exploratory increment

$\epsilon_{\min }$ minimum value of $\epsilon$

$\eta$ scale factor ${ }^{3}$ for $\epsilon$

$\theta$ vector determining projected point and subsequent $\delta$

$\kappa$ maximum number of random moves

$\rho$ scale factor for randomization ${ }^{3}$

$\phi$ current projected, exploratory or random point

$\phi_{\imath} \quad$ ith component of $\phi$

$\phi^{\circ}$ temporarily the best point

$\phi_{i}{ }^{\circ} \quad i$ th component of $\phi^{\circ}$.

The following variables are initially assigned values externally to the razor search package ${ }^{3}: k, \delta, \epsilon_{\min }, \eta, \kappa, \rho$, and $\phi^{\circ}$. The variables $j$ and $\kappa$ are common to the ripple search package.

Variables in PATSER as shown in Fig. 5: The variables $U_{\phi}, U_{\phi 0}, \phi$ and $\phi^{\circ}$ are defined as in RAZOR. The variables $\delta$, $\epsilon$ and $\theta$ are common to RAZOR, with $\alpha$ being the scale factor for reduction of $\delta$. The variable $\alpha$ is initially assigned a value externally to the razor search package.

Variables in EXPLR as shown in Fig. 6: The function $U$ and variables $S_{i}, U_{\phi}, U_{\phi 0}, \phi$ and $\phi_{\imath}$ are defined as in RAZOR. The variables $\kappa, \delta$ and $S$ are common to RAzOR.

Variables in PATMV as shown in Fig. 7: The function $U$ and variables $U_{\phi}, U_{\phi o}, \phi, \phi_{i}, \phi^{\circ}$ and $\phi_{i}{ }^{\circ}$ are defined as in RAZOR. The variables $k, \delta, \epsilon, \boldsymbol{\theta}$ and $S$ are common to RAZOR, with $m$ being the counter for projected points.

The Ripple Search Package

Variables in Function $\mathrm{U}$ as shown in Fig. 9:

$d$ dummy argument

$e$ end point of starting interval in Fibonacci search for local extremum

$i$ numbers intervals from left to right

$j$ integer set externally (initially 0 ) controlling reduction of $\sigma$

$j^{\prime}$ integer (initially 0 ) storing previous value of $j$

\footnotetext{
${ }^{3}$ In retrospect, the scale factors $\eta, \rho$, and $\zeta$ would probably be more conveniently calculated by the computer. They could be obtained by assigning initial values to $\epsilon$, the expression $\rho \cdot \epsilon$ in (7) and $\sigma$, say, $\epsilon_{1}, r_{1}$ and $\sigma_{1}$, respectively. Then $\eta \leftarrow\left(\epsilon_{1} / \epsilon_{\min }\right)^{1 / \kappa}, \rho \leftarrow r_{1} / \epsilon$, and $\zeta \leftarrow\left(\sigma_{1} / \sigma_{\min }\right)^{1 / \kappa}$.
} 
$k$ counter for ripple search

$k_{c}$ a uniform search is carried out whenever $k \equiv 1 \bmod k_{c}$ $n$ number of intervals in uniform search

$n_{f}$ value for $n$ in uniform search when $k \equiv 1 \bmod k_{c}$

$n_{\mathrm{o}}$ number of turning points plus one

o location of local extremum

$U$ maximum value of $y$

$x$ independent variable, e.g., frequency

$x_{l}$ lower bound on $x$ (left end)

$x_{u}$ upper bound on $x$ (right end)

$y$ objective function $y(x)$, e.g., network response deviation

$y_{l}$ value of $y$ at left hand $e$

$y_{0}$ local extremal value of $y$

$y_{r}$ value of $y$ at right hand $e$

$y_{t}$ temporary maximum value of $y$

$y_{u}$ value of $y$ at $x_{u}$

$\delta x$ increment of $x$ in uniform search

$\zeta$ scale factor ${ }^{3}$ for $\sigma$

$\kappa$ maximum value of $j$

$\sigma$ interval of uncertainty in Fibonacci search

$\sigma_{\min }$ minimum value of $\sigma$

$\tau=1(-1)$ if searching for maximum (minimum)

$\tau^{\prime}=1(-1)$ if the first turning point is expected to be a maximum (minimum).

The following variables are initially assigned values externally to the ripple search package ${ }^{3}: j, j^{\prime}, k_{c}, n, n_{f}, x_{l}, x_{u}$, $\zeta, \kappa$, and $\sigma_{\min }$. The variables $j$ and $\kappa$ are common to RAZOR. The variable $e$ is common to FIBSER.

Variables in LOCATE as shown in Fig. 10: The function $y$ and the variables $x, y_{t}$, and $U$ are defined as in the function subprogram $U$. The variables $e, n, n_{\circ}, o, x_{l}, x_{\psi}, \delta x$ and $\tau^{\prime}$ are common to $\mathrm{U}$.

$j=1(2)$ while searching for a local minimum (maximum)

$y_{x}$ current value of $y$ at $x$

$\check{y}$ temporary local minimum value of $y$

$\hat{y}$ temporary local maximum value of $y$.

\section{Computation of Reflection Coefficient}

Using the notation of Fig. 2, the basic steps in calculating the reflection coefficient $\rho$ of a resistively terminated cascade of transmission lines were

$$
\begin{aligned}
& Z_{I} \leftarrow R \\
& \theta \leftarrow \pi \bmod \left(2 f l_{i} / c, 1\right)\left|\theta-\frac{\pi}{2}\right| \leq 10^{-5} \\
& Z_{I} \leftarrow\left\{\begin{array}{ll}
Z_{2}{ }^{2} / Z_{I} & \\
Z_{i} \frac{Z_{I}+j Z_{i} \tan \theta}{Z_{i}+j Z_{I} \tan \theta} & \left|\theta-\frac{\pi}{2}\right|>10^{-5}
\end{array}\right\} \\
& \rho \leftarrow m, m-1, \cdots, 1 \\
& \qquad \frac{Z_{I}-1}{Z_{I}+1}
\end{aligned}
$$

where $j=\sqrt{-1}, c$ is the velocity of light and where $\bmod (a, b)$ is equivalent to $a-b$ times the integral part of $(a / b)$.

All programs were written in FORTRAN IV for the IBM $360 / 65$.

\section{ACKNOWLEDGMENT}

It is a pleasure to acknowledge many valuable discussions with Dr. A. Wexler of the Department of Electrical Engineering, University of Manitoba. The authors are also grateful for some helpful comments made by Dr. M. O'Hagan, SMU Institute of Technology, Dallas, Tex. The cooperation of the Institute for Computer Studies of the University of Manitoba is acknowledged.

\section{REFERENCES}

[1] L. S. Lasdon and A. D. Waren, "Optimal design of filters with bounded, lossy elements," IEEETrans. Circuit Theory, vol. CT-13, pp. 175-187, June 1966.

[2] A. D. Waren, L. S. Lasdon, and D. F. Suchman, "Optimization in engineering design," Proc. IEEE, vol. 55, pp. 1885-1897, November 1967.

[3] Y. Ishizaki and H. Watanabe, "An iterative Chebyshev approximation method for network design," IEEE Trans. Circuit Theory, vol. CT-15, pp. 326-336, December 1968.

[4] G. C. Temes and D. A. Calahan, "Computer-aided network optimization the state-of-the-art," Proc. IEEE, vol. 55, pp. 18321863, November 1967.

[5] G. C. Temes and D. Y. F. Zai, "Least pth approximation," IEEE Trans. Circuit Theory (Correspondence), vol. CT-16, pp. 235237, May 1969.

[6] R. Hooke and T. A. Jeeves, "' "Direct search' solution of numerical and statistical problems," J. ACM, vol. 8, pp. 212-229, April 1961 .

[7] W. T. Hatley, Jr., "Computer synthesis of wide-band impedancematching networks," Stanford Electronics Laboratories, Stanford University, Stanford, Calif., Tech. Rept. No. 6657-2, December 1967.

[8] J. W. Bandler, "Optimum noncommensurate stepped transmission-line transformers," Electronics Letters, vol. 4, pp. 212-213, May 31, 1968.

[9] J. W. Bandler and P. A. Macdonald, "Cascaded noncommensurate transmission-line networks as optimization problems," IEEE Trans. Circuit Theory (Correspondence), vol. CT-16, pp. 391-394, August 1969.

[10] J. W. Bandler, "Computer optimization of inhomogeneous waveguide transformers," this issue, pp. 563-571.

[11] - "Optimization methods for computer-aided design," this issue, pp. 533-552.

[12] I. M. Gelfand and M. L. Tsetlin, "The principle of nonlocal search in automatic optimization systems," Dokl. Akad. Nauk SSSR, vol. 137, pp. 295-298, March 1961 (English transl.: Sov. Phys. Dokl., vol. 6, pp. 192-194, September 1961).

[13] M. C. Pike, I. D. Hill, and F. D. James, "Note on Algorithm 2: Fibonacci search," Computer J., vol. 9, pp. 416-417, February 1967.

[14] D. J. Wilde and C. S. Beightler, Foundations of Optimization. Englewood Cliffs, N. J.: Prentice-Hall, 1967, ch. 6.

[15] G. L. Matthaei, L. Young, and E. M. T. Jones, Microwave Filters, Impedance Matching Networks, and Coupling Structures. New York: McGraw-Hill, 1964, ch. 6.

[16] A. F. Kaupe, Jr., "Algorithm 178: direct search," Commun. ACM, vol. 6, pp. 313-314, June 1963.

[17] M. Bell and M. C. Pike, "Remark on algorithm 178: direct search," Commun. ACM, vol. 9, pp. 684-685, September 1966.

[18] D. J. Wilde and C. S. Beightler [14], pp. 307-313.

[19] D. J. Wilde and C. S. Beightler [14], p. 312.

[20] H. H. Rosenbrock, "An automatic method for finding the greatest or least value of a function," Computer J., vol. 3, pp. 175-184, October 1960.

[21] M. J. D. Powell, "An efficient method for finding the minimum of a function of several variables without calculating derivatives," Computer J., vol. 7, pp. 155-162, July 1964.

[22] W. I. Zangwill, "Minimizing a function without calculating derivatives," Computer J., vol. 10, pp. 293-296, November 1967.

[23] F. E. Emery and M. O'Hagan, "Optimal design of matching networks for microwave transistor amplifiers," IEEE Trans. Microwave Theory and Techniques (Correspondence), vol. MTT-14, pp. 696-698, December 1966.

[24] M. O'Hagan, SMU Institute of Technology, Dallas, Tex., private communication. 